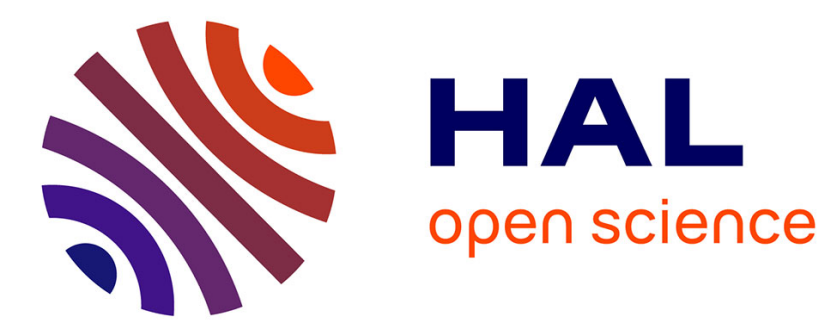

\title{
COOLING OR HEATING OF AN ATOMIC VAPOR USING COLLISIONALLY AIDED LASER EXCITATION
}

\author{
E. Giacobino, P. Berman, H. Stroke, O. Redi, M. Tawil
}

\section{- To cite this version:}

E. Giacobino, P. Berman, H. Stroke, O. Redi, M. Tawil. COOLING OR HEATING OF AN ATOMIC VAPOR USING COLLISIONALLY AIDED LASER EXCITATION. Journal de Physique Colloques, 1985, 46 (C1), pp.C1-275-C1-278. 10.1051/jphyscol:1985127 . jpa-00224500

HAL Id: jpa-00224500 https://hal.science/jpa-00224500

Submitted on 1 Jan 1985

HAL is a multi-disciplinary open access archive for the deposit and dissemination of scientific research documents, whether they are published or not. The documents may come from teaching and research institutions in France or abroad, or from public or private research centers.
L'archive ouverte pluridisciplinaire HAL, est destinée au dépôt et à la diffusion de documents scientifiques de niveau recherche, publiés ou non, émanant des établissements d'enseignement et de recherche français ou étrangers, des laboratoires publics ou privés. 


\title{
COOLING OR HEATING OF AN ATOMIC VAPOR USING COLLISIONALLY AIDED LASER EXCITATION
}

\author{
E. Giacobino, P.R. Berman*, H.H. Stroke*, O. Redi* and M. Tawi ${ }^{\star}$ \\ Laboratoire de Spectroscopie Hertzienne de l'ENS, Université Pierre et Marie \\ Curie, 75230 Paris Cedex 05, France \\ `Department of Physics, NYU, 4 Washington PLace, New-Yoxk, N.Y. 10003, U.S.A.
}

Résumé - Nous nous proposons, dans cet article, de considérer les collisions en présence d'un champ laser d'un point de vue un peu inhabituel, celui du bilan d'échange d'énergie entre le champ électromagnétique et les atomes.

Abstract - In this paper, we study the collisions in the presence of a laser field from a novel point of view, considering the exchange of energy between the electromagnetic field and the atoms.

\section{I - INTRODUCTION}

The idea of transferringmomentum or energy between the electromagnetic field and the external degrees of freedom of atoms or molecules has been the subject of a great deal of interest during the past few years. The proposed schemes can be separated in two classes, depending on whether the light field is near-resonant with the atoms or far from resonance.

The first technique can be interpreted in terms of radiation pressure : the atoms, when absorbing a photon, recoil with the momentum carried by the photon and can be slowed down by such repeated absorptions.

On the other hand, a different process has been proposed to slow down atoms : it relies on a difference in energy between the absorbed photon and the emitted photon, this difference being taken from the thermal energy of the atoms. Cooling by Raman scattering was hypothesized as early as 1950 by Kastler /1/. Since then other authors have proposed practical schemes to use this idea $/ 2 / / 3 /$. We shall see that this can only be achieved in the presence of collisional relaxation.

\section{II - PRINCIPLE}

We consider a collection of 2-level atoms subjected to non resonant laser irradiation of frequency $\Omega$; the detuning $\Delta$ from resonance is assumed to be larger that the Doppler width. Without collisions, if the laser is weak enough, energy conservation requires that emission of light has to occur at the Doppler shifted laser excitation frequency : this is Rayleigh scattering. The scattering process absorbs energy at a specific frequency inside the atomic bandwidth, and reemission starts from this point (we neglect here the recoil shift). In the presence of a strong laser irradiation higher order processes involving scattering at different frequencies may occur, but they conserve the sum internal atomic energy + field energy.

Let us now consider the case in which collisions may occur. We will concentrate on the collisionally aided radiative excitation (CARE) type of process, which can be described in a general way as

$\mathrm{A}_{1}+\mathrm{B}_{1}+\mathrm{h} \Omega \rightarrow \mathrm{A}_{2}+\mathrm{B}_{1}$

where $A_{j}$ and $B_{j}$ represent atoms of different species $A$ and $B$ in states $i$ and $j$, respectively.- Experimental studies to date have mainly concentrated on measurements of either CARE cross sections or the frequency distribution of the reemitted 1 ight. 
However, as implied in most recent treatments of light scattering /4/ this type of process also produces a change in the atomic velocities. The velocity changes can be viewed very easily : Let us consider radiation of frequency $\Omega$ acting on a twolevel system whose Bohr frequency is $\omega$ (with $\Omega-\omega=\Delta>$ Doppler width). As a result of the interaction with the field in the presence of a collision, the atom may absorb one photon from the input field and be excited to its upper state. In other words collisions provide a mechanism to redistribute the excited state population over the natural width of the upper level.

Since the final energy level of the atom is different from the energy of the absorbed photon, the energy difference must be compensated by a change in the translational energy of the colliding atoms.

As mentionned above, this process may have a potential use as a method for heating or cooling an atomic vapor. Tuning with $\Omega>\omega$ produces heating whereas that with $\Omega<\omega$ produces cooling.

\section{III - EXPERI MENT}

An experiment showing evidence for velocity changes in such processes has been carried out recently /5/. The experiment has been done with a blue detuning of the laser from the atomic frequency, that is in the case of heating. A change in the velocity distribution of the excited state atoms has been demonstrated. The vapor was subjected to laser irradiation detuned toward high frequencies from the $3 S-3 P$ transition in sodium (ground state to first excited state). The velocity distribution of the excited $3 \mathrm{P}$ sodium atoms was monitored by scanning a narrow-band probe laser through the Doppler-broadened resonance associated with absorption on another transition starting from the $3 P$ state $(3 P \rightarrow 4 D)$ (Fig. 1)

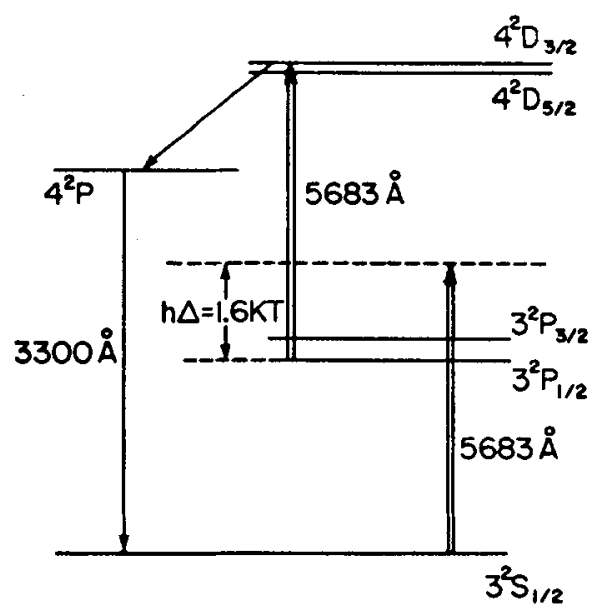

Fig. 1 - Sodium energy level diagram and excitation scheme. Actually the same laser is used for the two steps $3 S \rightarrow 3 P$ and $3 P \rightarrow 4 D$ (the scanning range around the $3 P \rightarrow 4 D$ resonance is negligible compared to $\Delta$ ).

The rare-gas used in our experiment were helium $(M=4)$, argon $(M=40)$ and xenon ( $M=$ 130). The excess energy $h \Delta=h(\Omega-\omega)$ is distributed between the two partners of the collision, sodium $(M=23)$ and rare gas according to the usual laws of classical mechanics. Consequently, we expect very small velocity changes for sodium in the helium-sodium collision, since the lighter helium atom carries off practically all the excess energy $h \Delta$. The velocity distribution of the $3 P$ excited sodium atom in that case will be essentially identical to the Maxwellian velocity distribution in the ground state; this provides a control measurement to which the velocity changes obtained in collision with the heavier rare gases, argon and xenon, can be compared. In the latter cases, the velocities of the 3P excited sodium atoms will be nonthermal and characterized by a nonequilibrium distribution which has been calcula ted using standard methods /6/. 
The experimental data (Table 1) show a significant increase of the widths of 3P - 4D probe resonances from helium to argon and xenon perturbers, thus demonstrating the velocity increase subsequent to the col1isiona11y aided excitation into the $3 \mathrm{P} 1$ eve1 (the widths include a contribution of the pressure and Iight-power broadenings on the probe transition, but these are the same for the three rare gases). Table I - Widths of the absorption resonance on the probe 3P-4D in GHz. The accuracy is about $0.1 \mathrm{GHz}$.

\begin{tabular}{ccccc}
\hline Rare Gas & Pressure (Torr) & $\mathrm{Na}-\mathrm{He}$ & $\mathrm{Na}-\mathrm{Ar}$ & $\mathrm{Na}$ \\
\hline 5 & 2.7 & 3.0 & 3.5 \\
10 & 2.7 & 3.2 & 4.0 \\
20 & 3.1 & 3.3 & \\
30 & 3.3 & 3.6 & \\
\hline
\end{tabular}

A coarse deconvolution of the experimental profile /5/ has allowed us to extract the magnitude of the Doppler width, and thus to get a measurement of the mean speed of the $3 \mathrm{P}$ atoms along the laser propagation axis. The results are shown in Table 2 along with theoretical predictions $/ 6 /$.

Table 2 - Comparison of the experimentally obtained widths with theory (power and pressure broadening extracted). All values are in $\mathrm{GHz}$.

\begin{tabular}{lrrr}
\hline Gas & Thermal Doppler width & $\begin{array}{c}\text { Theoretical width } \\
\text { including heating }\end{array}$ & $\begin{array}{c}\text { Experimental } \\
\text { Doppler width }\end{array}$ \\
\hline $\mathrm{Ar}$ & 1.8 & 2.9 & 2.4 \\
$\mathrm{Xe}$ & 1.8 & 3.5 & 2.8 \\
\hline
\end{tabular}

For both argon and xenon, it can be seen that the experimental values are significantly larger than the thermal Doppler widths. This gives evidence for the predicted "heating"effect. Nevertheless, the effect is smaller than theoretica11y calculated. One can account for this if one considers the processes that thermalize the atoms in the $3 \mathrm{P}$ state, in particular the velocity changing collisions and the radiation trapping.

\section{IV - EXPECTED COOLING OR HEATING}

The above experiment showed evidence for a change in the atomic velocities in the excited state; to actually produce a temperature change in the vapor, a large proportion of the atoms present in the vapor must undergo such a process.

Let us estimate the expected temperature change. The magnitude of the effect depends strongly on the detuning $\Delta=\Omega-\omega$ : each time an atom interacts with a photon, the energy $h \Delta$ is removed or added to the vapor. One may choose $h \Delta$ to be an appreciable fraction of $\mathrm{kT}$ (at $500 \mathrm{~K}, \mathrm{kT}=\mathrm{h} .10^{4} \mathrm{GHz}$ ), so the velocity changes are of the order of the r.m.s. velocity, that is a few $10^{2} \mathrm{~m} / \mathrm{sec}$ : they are large compared with those of order $1.0 \mathrm{~cm} / \mathrm{sec}$ which occur as a result of photon recoil.

The rate of excitation is given by :

$\frac{\mathrm{dn}}{\mathrm{dt}}=\frac{2 \chi^{2} \Gamma}{\Delta^{2}}$

where $\mathrm{n}$ is the population of the excited state and $\chi$ is the Rabi frequency. Then the rate at which the energy is exchanged with the laser is

$\frac{d W}{d t}=\frac{2 x^{2} \Gamma}{\Delta^{2}} h \Delta$

How the atom must decay by spontaneous emission, and $\mathrm{dn} / \mathrm{dt}$ is limited by the natu-

ral life-time. In the present case, if the vapor is dense enough, radiation trapping may occur, resulting in an effective lengthening of the excited state life-time. Subsequently the limit set to the maximum excitation rate may be lower than the natural decay rate by several orders of magnitude.

Nevertheless, one can calculate an order of magnitude of the cooling rate with experimental parameters meeting these requirements and reasonable cw laser powers ; one finds that the time scale at which thermal energy is removed or doubled is of order $10^{-1}$ sec. 
Now, as the experiment is done in a cell, we must consider the effect of the walls. The temperature gradient created between the laser beam and the walls of the cell depends on thermal diffusion properties; with usual thermal conductivities $\left(\sim 10^{-2} \mathrm{~J} / \mathrm{cm} . \mathrm{sec} .{ }^{\circ} \mathrm{K}\right.$ ) the expected temperature gradient is of order $1.0^{\circ} \mathrm{K} / \mathrm{cm}$ in the steady state. But in the considered cases, the thermal diffusion rate is slow ( $\sim 10 \mathrm{sec}^{-1}$ ) so significant transient temperature changes can be predicted with pulsed lasers, if the pulse is long enough. On the other hand it has been found /7/ that in rather dense (1 Torr) pure alkali vapors, the presence of numerous excited atoms diminishes the thermal conductivity, thus allowing to achieve steady state temperature gradients of order several $10^{\circ} \mathrm{K} / \mathrm{cm}$.

In conclusion, we have shown evidence for significant "heating" associated with the collisionally aided radiative excitation of the $3 P$ atoms using a laser detuned to the blue. We think that this process has a potential use for inducing macroscopic local temperature changes in a vapor, and could be a new method for heating or cooling the atoms.

REFERENCES

/1/ A. Kastler, J. Phys. Radium 11, (1950), 255.

$/ 2 /$ P.R. Berman and S. Stenholm, Opt. Commun. 24, (1978), 155.

/3/V.S. Letokhov, App1. Phys. 24, (1981), 119.

14/ J.L. Carlsten and A. Szöke, Phys. Rev. Lett. 36, (1976), 667; J.L. Carlsten, A. Szöke, and M.G. Raymer, Phys. Rev. A15, (1977), 1029.

/5/ E. Giacobino, M. Tawil, P.R. Berman, O. Redi and H.H. Stroke, Phys. Rev. A28, (1983), 2555 .

/6/ M. Tawil, Ph.D. Thesis (New-York University 1983) unpub1ished

/7/ E. Giacobino and P.R. Berman, NBS Special Publication n. 653, (1983), p.112. 